\title{
Desconhecimento sobre a campanha de vacinação contra o HPV entre estudantes brasileiros: uma análise multinível
}

\author{
Lack of awareness of the HPV vaccination campaign \\ among Brazilian students: a multilevel analysis
}

\begin{abstract}
Maria Aparecida Paulo dos Santos (https://orcid.org/0000-0002-6997-8168) ${ }^{1}$
Fábia Cheyenne Gomes de Morais Fernandes (https://orcid.org/0000-0002-0834-855X) ${ }^{1}$

Kenio Costa de Lima (https://orcid.org/0000-0002-5668-4398) ${ }^{1}$

Isabelle Ribeiro Barbosa (https://orcid.org/0000-0002-1385-2849) ${ }^{1}$
\end{abstract}

${ }^{1}$ Programa de PósGraduação em Saúde Coletiva, Faculdade de Ciências da Saúde do Trairi, Universidade Federal do Rio Grande do Norte. Av. Rio Branco 435, Centro. 59200-000 Santa Cruz RN Brasil. aparecida_cida4@ hotmail.com

\begin{abstract}
The scope of this article is to analyze the association between lack of awareness of the HPV vaccination campaign among Brazilian adolescents and individual and contextual factors. It involved a cross-sectional study with data from the National Student Health Survey (2015). Bivariate analysis was performed and Prevalence Ratios were calculated, in a multilevel Poisson Regression (95\%CI) to verify the effect of variables on the outcome. The outcome was significantly associated with being 15-19 years old ( $P R=1.36)$, studying during the afternoon/night shift $(P R=1.05)$, having already had sexual intercourse $(P R=1.10)$, with self-perceived poor or very poor health status $(P R=1.23)$, dissatisfied $(P R=1.14)$ or indifferent $(P R=1.15)$ regarding their body image, playing truant from classes without parental consent $(P R=1.10)$ and studying at a public school $(P R=1.24)$. There was less prevalence of the outcome among females $(P R=0.24)$ and in states with greater income inequality $(P R=0.80)$. The lack of awareness about the HPV vaccination campaign among adolescents was associated with individual characteristics, the context of the school and the unit of the federation. These findings indicate the importance of enhancing health promotion for vulnerable young people.
\end{abstract}

Key words Papillomavirus vaccines, Health promotion, Adolescent, Health inequalities, Multilevel analysis
Resumo O objetivo deste artigo é analisar a associação entre o desconhecimento sobre a campanha de vacinação contra o HPV entre adolescentes e fatores individuais e contextuais. Estudo transversal com dados da Pesquisa Nacional de Saúde do Escolar (2015). Foi realizada a análise bivariada e calculadas as Razões de Prevalência em uma Regressão de Poisson multinível (IC95\%) para verificar o efeito das variáveis no desfecho. $O$ desfecho esteve associado significativamente a ter 15-19 anos de idade $(R P=1,36)$, estudar no turno da tarde/noite $(R P=1,05)$, já ter tido relações sexuais $(R P=1,10)$, com autopercepção do estado de saúde ruim ou muito ruim $(R P=1,23)$, insatisfeito $(R P=1,14)$ ou indiferente $(R P=1,15) \grave{a}$ sua imagem corporal, que falta às aulas sem consentimento dos pais $(R P=1,10)$ e que estuda em escola pública $(R P=1,24)$. Houve menor prevalência do desfecho entre o sexo feminino $(R P=0,24)$ e em estados com maior desigualdade de renda $(R P=0,80)$. O desconhecimento sobre a campanha de vacinação contra o HPV entre adolescentes foi associado às características individuais e do contexto da escola e da unidade de federação. Esses achados indicam a importância de fortalecer a promoção à saúde voltada aos jovens em vulnerabilidade.

Palavras-chave Vacinas contra papilomavírus, Promoção da saúde, Adolescente, Desigualdades em saúde, Análise Multinível 


\section{Introdução}

O papilomavírus humano (HPV) é um vírus que infecta pele e mucosas (oral, genital e anal), principalmente através da transmissão sexual. Possui elevada prevalência em ambos os sexos, provocando verrugas anogenitais e até mesmo o câncer do colo do útero, vagina, vulva, pênis e ânus ${ }^{1,2}$.

As infecções por HPV são comuns em todo o mundo. A maior prevalência de HPV foi observada na África Subsaariana (24\%), Europa Oriental $(21,4 \%)$ e América Latina $(16,1 \%)$ e as mais baixa na América do Norte (4,7\%) e Ásia Ocidental $(1,7 \%)^{3}$. Na população brasileira, a prevalência de infecção por HPV é alta e varia de acordo com a localização anatômica do corpo, com taxas mais altas na região peniana (36\%), seguida pela região cervical e anal (aproximadamente $25 \%$ ) e depois pela região oral (12\%). Há também uma variação geográfica na prevalência do HPV no Brasil, com aumento da prevalência no Nordeste (exceto na região peniana) e escassos dados das regiões geográficas Norte e Centro-Oeste ${ }^{4}$.

Existem mais de cem sorotipos de HPV, sendo os sorotipos 16 e 18 classificados como HPV de alto risco para o câncer do colo do útero, estando relacionados à cerca de $70 \%$ dos $\operatorname{casos}^{1,2}$. O câncer de colo de útero ou câncer cervical, embora passível de prevenção e cura, é responsável por um grande número de morte entre mulheres, principalmente em países em desenvolvimento. É o segundo tipo de câncer mais frequente em mulheres que vivem em regiões menos desenvolvidas do mundo. Em 2018, foram registrados 570 mil novos casos deste câncer ( $84 \%$ dos novos casos no mundo) e 311 mil mulheres morreram de câncer do colo do útero; mais de $85 \%$ dessas mortes ocorreram em países de baixa e média renda $a^{2,5}$.

No Brasil, o Instituto Nacional de Câncer (INCA) estima que para cada ano do triênio 2020/2022 sejam diagnosticados 16.590 novos casos de câncer de colo do útero, com um risco estimado de 15,43 casos a cada 100 mil mulheres, ocupando a terceira posição de câncer no país ${ }^{6}$. Para a prevenção primária do câncer do colo do útero, é necessária a diminuição do risco de infecção pelo HPV, que está associada principalmente aos jovens com vida sexual ativa, considerando que metade das novas infecções ocorre entre pessoas nos três primeiros anos de atividade sexual ${ }^{7}$.

Em 2014, o Ministério da Saúde implementou no calendário de imunização a vacina tetravalente contra o HPV para meninas de 9 a 13 anos. A partir de 2017, o Ministério estendeu a vacinação para meninas de 9 a 14 anos e meninos de 11 a 14 anos. Essa vacina protege contra os tipos 6, 11, 16 e 18 do HPV ${ }^{8}$. Contudo, ainda é perceptível a falta de conhecimento de muitos adolescentes sobre a importância da vacina e que a mesma é disponibilizada de forma gratuita através do Sistema Único de Saúde (SUS), fazendo com que muitos deles mantenham a carteira de vacinação em atraso, seja por falta de orientação ou esquecimento?.

A adolescência é um período marcado por mudanças no desenvolvimento humano, indefinição e transição, acompanhadas de alterações fisiológicas, psicológicas e sociais. Ao longo dessas mudanças, é perceptível a ausência de orientações adequadas, seja pela família, escola ou sociedade, os tornando mais vulneráveis e menos sensíveis sobre a importância de se protegerem diante das doenças existentes e que podem ser preveníveis através da vacinação. Além disso, é notória a escassez de adolescentes buscando atendimento nas Unidades Básicas de Saúde, seja pela falta de interesse em cuidar de sua saúde, por se acharem saudáveis suficientes ou inocentemente acreditarem que não irão se contaminar ${ }^{10}$.

Destarte, uma forma de sensibilizar os adolescentes acerca da vacinação é através de ações de saúde nas escolas. Segundo Silva e Bodstein ${ }^{11}$, a escola historicamente representa espaço importante para práticas e vivências em saúde presentes nas relações entre os sujeitos envolvidos. Fatores determinantes das condições de saúde e doença podem ser problematizados no espaço escolar, como é o caso da vacinação contra o HPV. Para isto, o método educativo deve ser coletivo, de forma ampla, articulando escola, sistema de saúde e família, a fim de estabelecer seguimento na educação.

Em vista da importância dessa estratégia como prevenção ao câncer de colo do útero, torna-se imprescindível o monitoramento das ações relacionadas à saúde do escolar. A investigação do conhecimento da vacinação contra o HPV é de grande relevância para planejar e avaliar as políticas de saúde do adolescente, para assim compreender a necessidade de ações voltadas aos grupos de maior vulnerabilidade e risco.

O objetivo desse estudo foi analisar a associação entre o desconhecimento sobre a campanha de vacinação contra o HPV e fatores individuais e contextuais em adolescentes brasileiros incluídos na Pesquisa Nacional de Saúde do Escolar (PeNSE 2015). 


\section{Métodos}

Trata-se de um estudo de associação entre uma variável dependente que expressa o desconhecimento a respeito da campanha de vacinação contra o vírus HPV e variáveis independentes individuais e contextuais a partir de uma análise multinível. $\mathrm{O}$ primeiro nível correspondeu às variáveis individuais e o segundo e terceiro níveis correspondem às variáveis contextuais relativas às escolas e às Unidades da Federação, respectivamente.

Esse estudo utilizou dados secundários da amostra 01 do inquérito transversal da Pesquisa Nacional de Saúde do Escolar, 2015 (PeNSE 2015). A pesquisa foi realizada pelo Instituto Brasileiro de Geografia e Estatística (IBGE), em parceria com o Ministério da Saúde. A amostra de escolares do $9^{\circ}$ ano do Ensino Fundamental foi dimensionada de modo a estimar parâmetros populacionais (proporções ou prevalências) nos diversos domínios geográficos: cada uma das 26 capitais estaduais, mais o Distrito Federal, foi definida como um estrato geográfico; os demais municípios foram agrupados dentro de 26 estratos geográficos, representando cada um dos estados brasileiros, excluídas as capitais. Em cada um dos 53 estratos formados, foi dimensionada e selecionada uma amostra de escolas ${ }^{12-14}$.

Os tamanhos das amostras foram calculados para fornecer as estimativas em cada um dos estratos geográficos, com um erro máximo aproximado de $3 \%$, em valor absoluto, ao nível de confiança de $95 \%$ e considerando-se uma prevalência (proporção) da ordem de 0,5 (ou 50\%). A partir de uma amostragem complexa, foram selecionadas as escolas, no primeiro nível, e os estudantes, no segundo nível de seleção. Esses estudantes deveriam estar regularmente matriculados em escolas públicas e privadas das zonas urbana e rural de todo o território brasileiro, frequentando as aulas no ano da realização do inquérito. Sob essas condições, todos os estudantes das turmas das escolas sorteadas foram considerados elegíveis para participar do inquérito e convidados a responder ao questionário ${ }^{15}$. Participaram do inquérito 102.301 alunos matriculados no $9^{\circ}$ ano, em 3.040 escolas e 4.159 turmas em todo o país. Considerando os escolares matriculados e não respondentes, a perda amostral foi de cerca de $8,5 \%{ }^{16}$.

Para construção da variável dependente, denominada "Desconhecimento da campanha contra o HPV", foi selecionada a seguinte questão da pesquisa: "Você conhece/ouviu falar sobre a campanha de vacinação contra o vírus HPV?”. A variável dicotômica estava categorizada em “Sim” e "Não”. Das 102.301 respostas válidas ao questionário da PeNSE 2015, 10.538 indivíduos responderam que não conheciam a campanha de vacinação contra o HPV.

As variáveis explicativas foram agrupadas em três (3) blocos.

Bloco I - Características sociodemográficas e hábitos de vida, incluídas as variáveis: (i) Sexo (masculino e feminino); (ii) Cor da pele (Pretos, Pardos, Brancos e outros); (iii) Faixa etária (1114 anos, 14-19 anos); (iv) Morar com mãe e/ou pai - categorizada como Mora com os pais (escolares que residem com pai e mãe), Residem só com a mãe ou residem só com pai; ou Não mora com os pais; (v) Trabalha atualmente (sim, não); (vi) Possui acesso à Internet na residência (Sim, Não); (vii) ficou com fome por não ter comida suficiente em sua casa (sim, não); (viii) Uso do tabaco nos últimos 30 dias (sim, não); (ix) Uso do Álcool regular, ou uso nos últimos 30 dias (sim, não); (xiv) Drogas experimentação na vida (sim, não); (x) Turno em que estuda (manhã/intermediário; tarde/noite, integral).

Bloco II - Bloco de comportamentos e de saúde: (i) Ter tido Relação sexual (sim, não); (ii) agredido(a) fisicamente nos últimos 12 meses (sim, não); (iii) Autopercepção do estado de saúde (muito bom/bom, regular, ruim/muito ruim); (iv) Autopercepção da Imagem corporal (satisfeito, indiferente, insatisfeito); (v) O grau de escolaridade mais elevado que pretende concluir (até o ensino médio, ensino superior/pós-graduação); (vi) Falta às aulas ou à escola sem permissão dos seus pais ou responsáveis (sim, não); (vii) Na escola, você já recebeu orientação sobre prevenção de gravidez (sim, não); (viii) Na escola, você já recebeu orientação sobre AIDS ou outras Doenças Sexualmente Transmissíveis (DST) (sim, não); (ix) Na escola, você já recebeu orientação sobre como conseguir camisinha (preservativo) gratuitamente (sim, não); (x) Nos últimos 12 meses procurou algum serviço ou profissional de saúde para atendimento relacionado à própria saúde (sim, não).

Bloco III - variáveis contextuais da escola $e$ da Unidade da Federação: (i) Situação da Escola (urbana, rural); (ii) Dependência administrativa (pública, privada), (iii) Porte da Escola (pequena/médio porte - até 500 alunos, grande porte - >501 alunos); (iv) Esfera Administrativa da escola (federal, municipal, estadual, privada); (v) Existe Comitê de Saúde na Escola (sim, não); (vi) Adesão ao Programa Saúde na Escola (sim, não); 
(vii) A escola realiza ações conjuntas com Unidade Básica de Saúde ou Equipe de Saúde da Família ou Equipe de Atenção Básica (sim, não); (viii) Possui acesso à internet na escola (sim, não); (ix) Gini Estadual (0,490-0,560, 0,561-0,610, 0,611$0,650)$; (x) IDH Estadual $(0,732-0,824,0,666-$ $0,731,0,630-0,665)$, ambos categorizados em tercis.

As informações socioeconômicas por estado brasileiro foram extraídas do Programa das Nações Unidas para o Desenvolvimento a partir dos dados do Atlas do Desenvolvimento Humano do Brasil (http://atlasbrasil.org.br/2013/pt/consulta/), com informações resultantes do Censo demográfico Brasileiro de 2010, do Instituto Brasileiro de Geografia e Estatística (IBGE). A Figura 1 mostra o modelo conceitual proposto para a relação entre o desfecho e as variáveis independentes individuais e contextuais.

O efeito foi estimado através da Razão de Prevalências (RP) e seus respectivos intervalos de confiança (IC95\%) através da Regressão de Poisson multinível. Variáveis com associações significativas nas análises bivariadas $(p<0,20)$ foram ajustadas no modelo multivariado. $\mathrm{Na}$ análise multivariada, foram testados quatro modelos, considerando a Unidades da Federação no nível de agregação. No primeiro, o efeito contextual das UF's foi avaliado a partir de um modelo nulo usando o teste de razão de verossimilhança para verificar a significância e, posteriormente, três modelos foram testados: o primeiro apenas com o nível individual, o segundo com os níveis do indivíduo e da escola, e o modelo final, com os níveis individual e contextual da escola e das UF. Após o ajustamento, permaneceram no modelo final apenas as variáveis estatisticamente associadas $(\mathrm{p}<0,05)$. Mudanças na qualidade dos ajustes de cada modelo foram analisadas pelo teste da razão de verossimilhança e pelo percentual da variância explicada por cada modelo e seu respectivo intervalo de confiança. Para analisar os dados coletados utilizou-se o sofware Stata, versão 13 (Stata Corp., College Station, EUA).

Considerando a amostragem complexa aplicada para a seleção da amostra da PeNSE 2015, o peso amostral de cada participante foi obtido a partir das probabilidades de seleção do primeiro nível (escola) e do segundo nível (aluno), e este peso amostral de cada participante foi fornecido no banco final da pesquisa. Todavia, para a aplicação da análise multinível, são necessárias as probabilidades de seleção de cada nível, e estas informações não estão disponíveis no banco final da PeNSE 2015. Dessa forma, a análise multinível foi realizada considerando os comandos específicos para tal análise, sem utilizar os comandos da amostra complexa presente no Stata.

A PeNSE 2015 foi aprovada na Comissão Nacional de Ética em Pesquisa (CONEP), do Conselho Nacional de Saúde. O presente estudo utiliza dados secundários da PeNSE disponíveis em sites oficiais do Ministério da Saúde do Brasil, sendo dispensado de apreciação em comitê de ética em pesquisa, em conformidade com a Resolução ${ }^{\circ}$ 466/2012 do Conselho Nacional de Saúde.

\section{Resultados}

Nas Tabelas 1 a 3, encontram-se os resultados das análises bivariadas entre os desfechos e as variáveis independentes de nível individual e contextual. Observa-se que o desfecho "desconhecimento da campanha de vacinação contra o HPV" está associado significativamente ao sexo masculino, aos indivíduos de cor da pele preta, na faixa etária de 15 a 17 anos, sem acesso à internet no domicílio, que não mora com os pais, com hábitos de tabagismo, alcoolismo, uso de drogas e que relatam já ter sentido fome por falta de alimentação (Tabela 1). Esteve associado também à autopercepção da saúde ruim ou muito ruim, indiferente quanto à imagem corporal, com baixa expectativa de escolaridade (que pretendem concluir apenas o ensino médio, sem interesse em cursar ensino superior/pós-graduação), que sofreu agressão física, que não tiveram orientação sobre gravidez, camisinha e IST/AIDS na escola e que não procuraram pelos serviços de saúde (Tabela 2). A prevalência do desconhecimento da campanha de vacinação contra o HPV esteve associada também a estudar em escola pública, da esfera municipal e estadual, em escolas que não possuem comitê de saúde, e que não aderiram ao PSE, nem fazem ações conjuntas com a ESF, que não possuem acesso à internet e que estão localizadas em Unidades da Federação com baixo índice de Gini (Tabela 3).

$\mathrm{Na}$ primeira etapa da análise multinível, o desfecho foi testado em um modelo nulo para verificar o efeito contextual. Na Tabela 4, é possível observar que houve efeito contextual em nível de unidade da federação, já que o valor da Razão de verossimilhança foi significativo, mostrando a adequação da amostra para a realização da análise. A segunda etapa foi a realização do modelo multinível incluindo somente o nível individual. No modelo 1, as variáveis "trabalha atualmente", "alcoolismo", "tabagismo", "uso de drogas" e 
Nível 3 - Contexto da Unidade Federativa

Índice de Gini

Índice de Desenvolvimento Humano

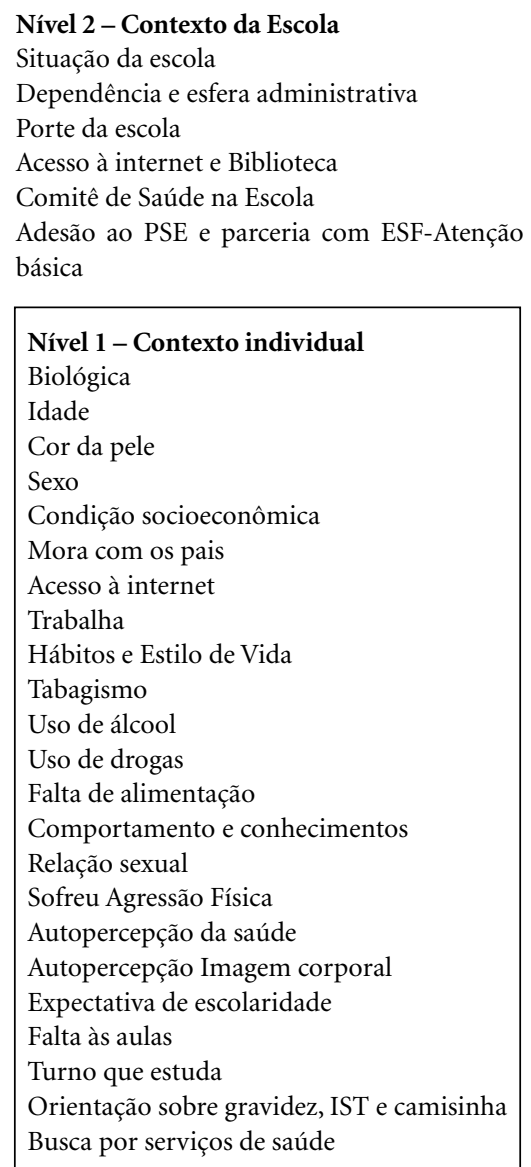

Figura 1. Fonte de dados, variáveis e níveis de análise empregados no modelo de Regressão de Poisson multinível.

Fonte: Instituto Brasileiro de Geografia e Estatística (IBGE) e Pesquisa Nacional de Saúde do Escolar, 2015.

"morar com os pais" perderam significância estatística ou apresentaram colinearidade e, portanto, foram excluídas do modelo. A análise das Razões de prevalências, nesta etapa, mostrou que algumas características estão mais fortemente associadas ao desfecho: faixa etária de 15 a 19 anos ( $\mathrm{RP}=1,39$; IC95\%: 1,32-1,44), menor expectativa de escolaridade ( $\mathrm{RP}=1,34$; IC95\%: 1,28-1,39), que não recebeu orientação sobre IST $(\mathrm{RP}=1,52$; IC95\%: 1,43-1,59), não recebeu orientação sobre camisinha ( $\mathrm{RP}=1,35$; IC95\%: 1,21-1,41) e que não recebeu orientação sobre gravidez $(\mathrm{RP}=1,28$; IC95\%: 1,22-1,35). A Razão de verossimilhança observada para o modelo 1 em relação ao modelo nulo variou de 180,33 para 171,71 , indicando um importante efeito das Unidades da federação no desfecho e sobre as variáveis individuais.

No modelo 2, foram incluídas as variáveis de contexto escolar. As variáveis "comitê de saúde na escola" ( $\mathrm{p}=0,894)$, "adesão ao PSE" ( $\mathrm{p}=0,826)$, acesso à internet $(\mathrm{p}=0,510)$ e esfera administrativa $(\mathrm{p}=0,070)$ perderam significância estatística e foram excluídas do modelo (Tabela 4). No modelo 2, todas as variáveis individuais permaneceram significativas; a única variável do contexto da escola mantida foi a "dependência administrativa”, mostrando que nas escolas públicas há maior prevalência do desconhecimento sobre a vacinação contra o HPV (RP=1,17; IC95\%: 1,101,25). A Razão de verossimilhança observada 
Tabela 1. Desconhecimento sobre a campanha de vacinação contra o HPV e sua associação com variáveis independentes sociodemográficas e de hábitos de vida entre adolescentes brasileiros. PeNSE (2015).

\begin{tabular}{|c|c|c|c|c|c|}
\hline Variáveis & $\begin{array}{c}\text { Prevalência \% } \\
(\mathrm{N})\end{array}$ & p-valor & $\mathbf{R P}_{\text {bruta }}$ & IC95\% & p-valor \\
\hline \multicolumn{6}{|l|}{ Sexo } \\
\hline Masculino & $18,19(8.407)$ & & 1 & & \\
\hline Feminino & $4,23(2.131)$ & $<0,001$ & 0,23 & $0,22-0,24$ & $<0,001$ \\
\hline \multicolumn{6}{|l|}{ Cor da pele } \\
\hline Brancos e outros & $11,13(4.452)$ & & 1 & & \\
\hline Pardos & $10,04(4.477)$ & $<0,001$ & 0,91 & $0,88-0,95$ & $<0,001$ \\
\hline Pretos & $13,36(1.609)$ & & 1,22 & $1,15-1,29$ & $<0,001$ \\
\hline \multicolumn{6}{|l|}{ Faixa etária } \\
\hline 11-14 anos & $8,88(5.838)$ & & 1 & & \\
\hline 15-19 anos & $15,22(4.700)$ & $<0,001$ & 1,77 & $1,70-1,84$ & $<0,001$ \\
\hline \multicolumn{6}{|l|}{ Fome } \\
\hline Não & $10,59(9.082)$ & & 1 & & \\
\hline Sim & $13,36(1.456)$ & $<0,001$ & 1,26 & $1,19-1,33$ & $<0,001$ \\
\hline \multicolumn{6}{|l|}{ Trabalha atualmente } \\
\hline Não & $10,31(8.729)$ & & 1 & & \\
\hline Sim & $15,09(8.729)$ & $<0,001$ & 1,44 & $1,37-1,52$ & $<0,001$ \\
\hline \multicolumn{6}{|l|}{ Tabagismo } \\
\hline Não & $10,67(9.783)$ & & 1 & & \\
\hline Sim & $15,18(755)$ & $<0,001$ & 1,41 & $1,31-1,52$ & $<0,001$ \\
\hline \multicolumn{6}{|l|}{ Alcoolismo } \\
\hline Não & $10,64(7.768)$ & & 1 & & \\
\hline Sim & $11,74(2.770)$ & $<0,001$ & 1,09 & $1,04-1,14$ & $<0,001$ \\
\hline \multicolumn{6}{|l|}{ Drogas } \\
\hline Não & $10,55(9.342)$ & & 1 & & \\
\hline Sim & $14,79(1.196)$ & $<0,001$ & 1,40 & $1,31-1,48$ & $<0,001$ \\
\hline \multicolumn{6}{|l|}{ Mora com os pais } \\
\hline Mora com os pais & $10,88(9.825)$ & & 1 & & \\
\hline Não mora com os pais & $11,19(698)$ & 0,035 & 1,03 & $0,95-1,11$ & 0,372 \\
\hline Mora com a mãe ou com o pai & $19,74(15)$ & & 1,82 & $1,10-3,01$ & 0,020 \\
\hline \multicolumn{6}{|l|}{ Acesso Internet } \\
\hline Sim & $10,46(7.814)$ & & 1 & & \\
\hline Não & $12,43(2.724)$ & $<0,001$ & 1,23 & $1,17-1,28$ & $<0,001$ \\
\hline \multicolumn{6}{|l|}{ Turno que estuda } \\
\hline Manhã/intermediário & $10,52(6.303)$ & & 1 & & \\
\hline Tarde/noite & $11,57(4.057)$ & $<0,001$ & 1,14 & $1,10-1,19$ & $<0,001$ \\
\hline Integral & $10,93(178)$ & & 1,09 & $0,94-1,27$ & 0,243 \\
\hline
\end{tabular}

Fonte: Elaborado pelos autores.

para o modelo 2 em relação ao modelo 1 variou de 171,71 para 157,83 , mostrando a adequação da análise à modelagem multinível.

No modelo final, com a inclusão da característica das Unidades da Federação, o desconhecimento da campanha de vacinação sobre o HPV esteve associado significativamente a pertencer à faixa etária de 15-19 anos de idade $(\mathrm{RP}=1,36)$, ter fome por não ter alimento $(\mathrm{RP}=1,10)$, estudar no turno da tarde/noite $(\mathrm{RP}=1,05)$ e in- tegral $(\mathrm{RP}=1,11)$, já ter tido relações sexuais $(\mathrm{RP}=1,10)$, ter sofrido agressão física $(\mathrm{RP}=1,21)$, com autopercepção do estado de saúde regular $(\mathrm{RP}=1,05)$, ruim ou muito ruim $(\mathrm{RP}=1,23)$, insatisfeito $(\mathrm{RP}=1,14)$ ou indiferente $(\mathrm{RP}=1,15)$ à sua imagem corporal, que falta às aulas sem consentimento dos pais $(\mathrm{RP}=1,10)$, que não recebeu orientação sobre gravidez $(\mathrm{RP}=1,28)$, sobre IST $(\mathrm{RP}=1,51)$ e sobre camisinha $(\mathrm{RP}=1,38)$ e que estuda em escola pública $(\mathrm{RP}=1,24)$. Pertencer ao 
Tabela 2. Desconhecimento sobre a campanha de vacinação contra o HPV e sua associação com variáveis independentes de comportamento e de saúde entre adolescentes brasileiros. PeNSE (2015).

\begin{tabular}{|c|c|c|c|c|c|}
\hline Variáveis & $\begin{array}{c}\text { Prevalência \% } \\
(\mathrm{N})\end{array}$ & p-valor & $\mathbf{R} \mathbf{P}_{\text {bruta }}$ & IC95\% & p-valor \\
\hline \multicolumn{6}{|l|}{ Relação Sexual } \\
\hline Não & $9,37(6.551)$ & & 1 & & \\
\hline Sim & $14,92(3.987)$ & $<0,001$ & 1,61 & $1,55-1,68$ & $<0,001$ \\
\hline \multicolumn{6}{|l|}{ Agressão Física } \\
\hline Não & $10,77(10.210)$ & & 1 & & \\
\hline Sim & $17,60(328)$ & $<0,001$ & 1,61 & $1,44-1,80$ & $<0,001$ \\
\hline \multicolumn{6}{|l|}{ Autopercepção da saúde } \\
\hline Muito bom/Bom & $10,91(7.532)$ & & 1 & & \\
\hline Regular & $9,8(1.968)$ & $<0,001$ & 0,90 & $0,86-0,95$ & $<0,001$ \\
\hline Ruim/Muito Ruim & $13,87(1.038)$ & & 1,27 & $1,19-1,36$ & $<0,001$ \\
\hline \multicolumn{6}{|l|}{ Autopercepção Imagem corporal } \\
\hline Satisfeito & $11,01(7.614)$ & & 1 & & \\
\hline Indiferente & $12,04(1.231)$ & $<0,001$ & 1,08 & $1,02-1,15$ & 0,009 \\
\hline Insatisfeito & $9,81(1.693)$ & & 0,88 & $0,83-0,93$ & $<0,001$ \\
\hline \multicolumn{6}{|l|}{ Expectativa de escolaridade } \\
\hline Ensino superior/pós-graduação & $8,60(4.853)$ & & 1 & & \\
\hline Até o Ensino médio & $14,15(5.685)$ & $<0,001$ & 1,64 & $1,58-1,71$ & $<0,001$ \\
\hline \multicolumn{6}{|l|}{ Falta às aulas } \\
\hline Não & $10,13(7.745)$ & & 1 & & \\
\hline Sim & $13,83(10.538)$ & $<0,001$ & 1,35 & $1,21-1,41$ & $<0,001$ \\
\hline \multicolumn{6}{|l|}{ Orientação sobre Gravidez } \\
\hline $\operatorname{Sim}$ & $8,90(6.764)$ & & 1 & & \\
\hline Não & $18,31(3.774)$ & $<0,001$ & 2,04 & $1,96-2,13$ & $<0,001$ \\
\hline \multicolumn{6}{|l|}{ Orientação sobre camisinha } \\
\hline Sim & $8,99(5.839)$ & & 1 & & \\
\hline Não & $14,82(4.699)$ & $<0,001$ & 1,65 & $1,58-1,71$ & $<0,001$ \\
\hline \multicolumn{6}{|l|}{ Orientação sobre DST e AIDS } \\
\hline $\operatorname{Sim}$ & $9,25(7.769)$ & & 1 & & \\
\hline Não & 21,91 (2.769) & $<0,001$ & 2,35 & $2,25-2,46$ & $<0,001$ \\
\hline \multicolumn{6}{|l|}{ Busca por serviços de saúde } \\
\hline $\operatorname{Sim}$ & $8,78(4.743)$ & $<0,001$ & 0,64 & $0,61-0,66$ & $<0,001$ \\
\hline Não & $13,60(5.795)$ & & 1 & & \\
\hline
\end{tabular}

Fonte: Elaborado pelos autores.

sexo feminino $(\mathrm{RP}=0,24)$ e viver em estados com maior desigualdade de renda $(\mathrm{RP}=0,80)$ foram fatores associados à menor prevalência do desfecho. A Razão de verossimilhança observada para o modelo final em relação ao modelo 2 variou de 157,83 para 91,25 , mostrando a adequação da análise à modelagem multinível.

\section{Discussão}

O presente estudo evidenciou que o desconhecimento sobre da campanha de vacinação contra o HPV entre os adolescentes avaliados na PENSE está associado tanto a características individuais quanto do contexto da escola e da unidade da federação de residência.

A desinformação dos adolescentes quanto à vacinação contra o HPV foi também destacada na pesquisa de Viegas et al. ${ }^{9}$ que entrevistou alunos do $9^{\circ}$ ano do ensino fundamental em Minas Gerais, os quais questionavam "preciso mesmo tomar vacina?", pela indecisão frente à vacinação e à ansiedade. Os resultados identificaram a cobertura vacinal de apenas $45,1 \%$ para todas as vacinas do calendário nacional do adolescente, sendo que apenas $5 \%$ relataram uso da vacina contra o HPV 9 .

Outro fator responsável pela baixa adesão à vacinação é o desconhecimento sobre a seguran- 
Tabela 3. Desconhecimento sobre a campanha de vacinação contra o HPV e associação com caraterísticas das escolas e da Unidade da Federação.

\begin{tabular}{|c|c|c|c|c|c|}
\hline Variáveis & $\begin{array}{c}\text { Prevalência \% } \\
(\mathrm{N})\end{array}$ & p-valor & $\mathbf{R} \mathbf{P}_{\text {bruta }}$ & IC95\% & p-valor \\
\hline \multicolumn{6}{|l|}{ Características da escola } \\
\hline \multicolumn{6}{|l|}{ Situação da Escola } \\
\hline Urbana & $10,88(9685)$ & & 1 & & \\
\hline Rural & $11,17(853)$ & 0,444 & 1,07 & $1,00-1,15$ & 0,038 \\
\hline \multicolumn{6}{|l|}{ Dependência administrativa } \\
\hline Privada & $8,35(1679)$ & & 1 & & \\
\hline Pública & $11,58(8859)$ & $<0,001$ & 1,40 & $1,33-1,48$ & $<0,001$ \\
\hline \multicolumn{6}{|l|}{ Porte da Escola } \\
\hline Pequena/Médio porte & $10,87(2736)$ & & 1 & & \\
\hline Grande Porte & $10,92(7802)$ & 0,851 & 0,98 & $0,94-1,03$ & 0,643 \\
\hline \multicolumn{6}{|l|}{ Esfera administrativa } \\
\hline Privada & $8,35(1679)$ & & 1 & & \\
\hline Municipal & $10,68(3192)$ & $<0,001$ & 1,3 & $1,22-1,38$ & $<0,001$ \\
\hline Estadual & $12,18(5641)$ & & 1,47 & $1,39-1,56$ & $<0,001$ \\
\hline Federal & $9,15(26)$ & & 1,20 & $0,81-1,78$ & 0,343 \\
\hline \multicolumn{6}{|l|}{ Comitê de Saúde na Escola } \\
\hline Sim & $10,63(3704)$ & & 1 & & \\
\hline Não & $11,06(6834)$ & 0,042 & 1,035 & $0,99-1,078$ & 0,094 \\
\hline \multicolumn{6}{|l|}{ Adesão ao PSE } \\
\hline Sim & $10,94(5118)$ & & 1 & & \\
\hline Não & $10,87(5420)$ & 0,740 & 1 & $0,92-1,00$ & 0,058 \\
\hline \multicolumn{6}{|l|}{ Ações parceria com a ESF } \\
\hline Sim & $10,90(7064)$ & & 1 & & \\
\hline Não & $10,92(3474)$ & 0,947 & 1 & $0,93-1,01$ & 0,236 \\
\hline \multicolumn{6}{|l|}{ Acesso à internet na escola } \\
\hline Sim & $10,72(7576)$ & & & & \\
\hline Não & $11,41(2962)$ & 0,002 & 1 & $1,04-1,14$ & $<0,001$ \\
\hline \multicolumn{6}{|c|}{ Características da Unidade Federativa } \\
\hline \multicolumn{6}{|l|}{ Gini Estadual } \\
\hline $0,490-0,560$ & $11,85(4306)$ & & 1 & & \\
\hline $0,561-0,610$ & $10,67(2820)$ & $<0,001$ & 0,90 & $0,78-1,04$ & 0,161 \\
\hline $0,611-0,650$ & $1007(3124)$ & & 0,84 & $0,73-0,95$ & 0,008 \\
\hline \multicolumn{6}{|l|}{ IDH Estadual } \\
\hline $0,732-0,824$ & $11,12(3175)$ & & 1 & & \\
\hline $0,666-0,731$ & $11,45(4439)$ & $<0,001$ & 1,03 & $0,89-1,19$ & 0,648 \\
\hline $0,630-0,665$ & $9,98(2924)$ & & 0,90 & $0,77-1,15$ & 0,204 \\
\hline
\end{tabular}

Fonte: Elaborado pelos autores.

ça e efetividade da vacina ofertada, bem como os tabus existentes na sociedade. Silva e Bodstein ${ }^{11}$, evidenciaram em seu estudo que os pais deixam de vacinar suas filhas devido à falta de discussão sobre sexualidade. O receio de que as meninas vacinadas adotem comportamentos sexuais arriscados ou que se tornem sexualmente ativas, constituem barreiras à imunização contra o HPV ${ }^{11}$.

O interesse sobre sexualidade, na fase da adolescência, gera muita curiosidade e o contato com as informações adequadas apresentam repercussões positivas. Furlanetto et al. ${ }^{17}$ mostraram que os adolescentes se sentiam mais confortáveis para conversar ou tirar dúvidas sobre sexo e sexualidade com a família, mas havia dificuldades em estabelecer um diálogo. O sentimento de confiança também foi apontado como relevante para que se pudesse estabelecer um diálogo franco com os profissionais da escola, mas o receio da exposição íntima e a possibilidade de conflito com os fami- 
Tabela 4. Associação multinível entre o desconhecimento de adolescentes brasileiros sobre a campanha de vacinação contra o HPV e características individuais e contextuais.

\begin{tabular}{|c|c|c|c|c|c|c|c|c|c|c|}
\hline & Modelo Nulo & \multicolumn{3}{|c|}{ Modelo 1} & \multicolumn{3}{|c|}{ Modelo 2} & \multicolumn{3}{|c|}{ Modelo Final } \\
\hline & $\mathrm{RP}_{\mathrm{aj}} \quad \mathrm{IC} 95 \%$ & $\mathbf{R P} \mathbf{P a j}_{\mathbf{a j}}$ & IC95\% & p-valor & $\mathbf{R} \mathbf{P}_{\mathrm{aj}}$ & IC95\% & p-valor & $\mathbf{R P}_{\mathrm{aj}}$ & IC95\% & p-valor \\
\hline \multicolumn{11}{|l|}{ Nível Individual } \\
\hline Sexo Feminino & & 0,25 & $0,23-0,26$ & $<0,001$ & 0,24 & $0,23-0,26$ & $<0,001$ & 0,24 & $0,23-0,26$ & $<0,001$ \\
\hline Faixa etária (15-19 anos) & & 1,39 & $1,32-1,44$ & $<0,01$ & 1,36 & $1,30-1,42$ & $<0,001$ & 1,36 & $1,30-1,42$ & $<0,001$ \\
\hline $\begin{array}{l}\text { Ter Fome por não ter } \\
\text { alimento }\end{array}$ & & 1,10 & $1,03-1,16$ & 0,001 & 1,10 & $1,04-1,17$ & 0,002 & 1,10 & $1,04-1,17$ & 0,002 \\
\hline $\begin{array}{l}\text { Estudar no turno da Tarde/ } \\
\text { Noite }\end{array}$ & & 1,09 & $1,05-1,14$ & $<0,001$ & 1,06 & $1,01-1,10$ & 0,008 & 1,05 & $1,01-1,10$ & 0,007 \\
\hline Estudar no turno Integral & & 1,12 & $0,97-1,31$ & 0,116 & 1,10 & $0,94-1,28$ & 0,194 & 1,11 & $0,95-1,29$ & 0,173 \\
\hline Relação Sexual & & 1,10 & $1,05-1,15$ & $<0,001$ & 1,09 & $1,05-1,14$ & $<0,001$ & 1,10 & $1,05-1,14$ & $<0,001$ \\
\hline Sofreu Agressão Física & & 1,20 & $1,07-1,34$ & 0,001 & 1,21 & $1,09-1,36$ & $<0,001$ & 1,21 & $1,08-1,36$ & $<0,001$ \\
\hline $\begin{array}{l}\text { Autopercepção Estado de } \\
\text { Saúde Regular }\end{array}$ & & 1,05 & $0,99-1,10$ & 0,063 & 1,05 & 0,99-1,09 & 0,055 & 1,05 & $1,00-1,10$ & $<0,001$ \\
\hline $\begin{array}{l}\text { Autopercepção Estado de } \\
\text { Saúde Ruim/Muito Ruim }\end{array}$ & & 1,23 & $1,15-1,31$ & 0,001 & 1,23 & $1,15-1,31$ & $<0,001$ & 1,23 & $1,15-1,32$ & $<0,001$ \\
\hline $\begin{array}{l}\text { Avaliação Imagem } \\
\text { Corporal Indiferente }\end{array}$ & & 1,13 & $1,07-1,21$ & $<0,001$ & 1,15 & $1,08-1,22$ & $<0,001$ & 1,15 & $1,08-1,22$ & $<0,001$ \\
\hline $\begin{array}{l}\text { Avaliação Imagem } \\
\text { Corporal Insatisfeito }\end{array}$ & & 1,13 & $1,06-1,18$ & $<0,001$ & 1,14 & $1,08-1,21$ & $<0,001$ & 1,14 & $1,08-1,21$ & $<0,001$ \\
\hline $\begin{array}{l}\text { Expectativa de } \\
\text { Escolaridade }\end{array}$ & & 1,34 & $1,28-1,39$ & $<0,001$ & 1,33 & $1,28-1,38$ & $<0,001$ & 1,32 & $1,27-1,38$ & $<0,001$ \\
\hline Falta às aulas & & 1,11 & $1,06-1,16$ & $<0,001$ & 1,10 & $1,05-1,15$ & $<0,001$ & 1,10 & $1,05-1,15$ & $<0,001$ \\
\hline $\begin{array}{l}\text { Não recebeu orientação } \\
\text { sobre gravidez }\end{array}$ & & 1,28 & $1,22-1,35$ & $<0,001$ & 1,28 & $1,22-1,34$ & $<0,001$ & 1,28 & $1,22-1,35$ & $<0,001$ \\
\hline $\begin{array}{l}\text { Não recebeu orientação } \\
\text { sobre IST/AIDS }\end{array}$ & & 1,52 & $1,43-1,59$ & $<0,001$ & 1,51 & $1,43-1,59$ & $<0,001$ & 1,51 & $1,43-1,59$ & $<0,001$ \\
\hline $\begin{array}{l}\text { Não recebeu orientação } \\
\text { sobre camisinha }\end{array}$ & & 1,35 & $1,21-1,41$ & $<0,001$ & 1,38 & $1,32-1,44$ & $<0,001$ & 1,38 & $1,32-1,44$ & $<0,001$ \\
\hline \multicolumn{11}{|l|}{ Nível Escola } \\
\hline $\begin{array}{l}\text { Dependência } \\
\text { administrativa tipo pública }\end{array}$ & & & & & 1,17 & $1,10-1,25$ & $<0,001$ & 1,24 & $1,17-1,31$ & $<0,001$ \\
\hline \multicolumn{11}{|l|}{ Nível Unidade da Federação } \\
\hline \multicolumn{11}{|l|}{ Gini } \\
\hline $0,490-0,560$ & & & & & & & & 1 & & \\
\hline $0,561-0,610$ & & & & & & & & 0,85 & $0,75-0,97$ & 0,018 \\
\hline $0,611-0,650$ & & & & & & & & 0,80 & $0,71-0,91$ & $<0,001$ \\
\hline \multicolumn{11}{|l|}{ Random effects } \\
\hline Variância $(95 \% \mathrm{CI})$ & $\begin{array}{c}0,0245 \\
(0,013-0,044)\end{array}$ & 0,0 & $239(0,013-$ & $-0,043)$ & & $022(0,012-($ & $0,042)$ & & $148(0,007$ & $0,027)$ \\
\hline LR test ( $\mathrm{p}$-value) & $\begin{array}{c}180,33 \\
(<0,001)\end{array}$ & & $171,71(<0$, & 001) & & $157,83(<0$, & 001) & & $91,25(<0,0$ & 01) \\
\hline
\end{tabular}

$\mathrm{RP}_{\mathrm{aj}}$ : razão de prevalência ajustada; IC95\%: intervalo de confiança 95\%; p-valor: valor de p; LR test: Likelihood ratio.

Fonte: Elaborado pelos autores.

liares foram os principais motivos pelos quais os adolescentes não participavam de discussões ${ }^{17}$.

Neste sentido, a escola pode ser reconhecida como um espaço potencial para ampliar e garantir a saúde do escolar. O Programa Saúde na Escola foi instituído em 2007, como uma proposta dos Ministérios da Saúde e da Educação, com a finalidade de colaborar, mediante ações de prevenção e promoção de saúde, atuando também em vulnerabilidades que possam comprometer o desenvolvimento escolar dos estudantes. Os profissionais de saúde, então, se responsabilizam 
pelo empoderamento de estudantes, professores e funcionários das escolas, estabelecendo um vínculo que possibilite o acesso às necessidades das crianças e adolescentes ${ }^{18}$. Destacamos que o Programa Nacional de Imunizações (PNI) tem promovido inúmeros avanços e sua atuação tem contribuído para o sucesso no controle de doenças imunopreveníveis no Brasil.

Outro fator preocupante é a maior prevalência do desconhecimento entre os adolescentes do sexo masculino. Estudos apontam que o vírus do HPV pode ser repassado mais facilmente do homem para a mulher. Além da não adesão à vacinação, existe a resistência por parte dos homens ao uso dos preservativos, masculinos e femininos. Mesmo os preservativos protegendo parcialmente da contaminação, devido a possibilidade do contato com lesões que fiquem descobertas, eles são importantes para evitar o contágio durante a penetração ${ }^{11}$. Um estudo que utilizou um modelo matemático para avaliar estratégias de vacinação no controle da transmissão do HPV na população humana, mostrou que a não inclusão da vacinação de homens só podem controlar a doença se mais de $90 \%$ das mulheres forem vacinadas ${ }^{19}$.

O presente estudo mostrou também que o não conhecimento da campanha de vacinação sobre o HPV também esteve associado a comportamentos sexuais de risco e ausência de orientação. Um estudo nacional mostrou que houve uma redução na prevalência do uso de preservativo, de $75,9 \%$ em 2009 para $66,2 \%$ em 2015, redução da orientação para prevenção de gravidez nas escolas públicas, de $81,1 \%$ para $79,3 \%$ e de preservativo gratuito nas escolas privadas, de $65,4 \%$ para $57,3 \%$. Observou-se que meninos iniciaram a vida sexual mais precocemente, com maior número de parceiros e menor uso de preservativo, bem como receberam menor orientação sobre prevenção desses eventos do que as meninas ${ }^{20}$.

Entre os hábitos de risco, o uso do álcool, tabaco e outras drogas foram associados ao desconhecimento sobre a vacina. $O$ tabaco é a segunda droga mais consumida entre jovens no mundo. Desta forma, é importante a realização de ações conjuntas de promoção e prevenção contra o HPV e o tabagismo, visto que o tabaco também é cofator no desenvolvimento do câncer de colo do útero e interfere na imunidade. Em relação às demais drogas, estas podem proporcionar comportamentos que podem levar a situações de vulnerabilidade para o contágio ${ }^{21}$.

A maior prevalência de desconhecimento associada à baixa expectativa de escolaridade entre os entrevistados da PENSE corrobora com outros achados na literatura. Abreu ${ }^{22}$ traz em seu estudo que pessoas com um maior grau de instrução, apresentam maior preocupação quanto a prevenção de IST's quando comparadas a pessoas com menor nível educacional. Isso pode ser justificado pelo fato de que indivíduos com mais tempo de estudo compreendem melhor os conteúdos apresentados, bem como possuem maior interesse em obter novos conhecimentos e buscar fontes confiáveis de informação, como a literatura científica ${ }^{22}$.

Outro ponto destacado nesse estudo é o desconhecimento das campanhas de vacinação entre os jovens que relataram não ter comida suficiente, usado nesse estudo como uma proxy de condição socioeconômica. A escolaridade e o fator econômico interferem significativamente na adesão às ações de promoção da saúde. Um estudo realizado em Campinas-SP, em 2011, apontou que 46\% das pessoas entrevistadas que pertenciam aos estratos socioeconômicos A e B responderam ter ouvido falar sobre o HPV, enquanto apenas $33,1 \%$ das classes $\mathrm{C}$ e D relataram possuir conhecimento sobre esse assunto ${ }^{22}$. Todavia, a menor prevalência do desconhecimento da vacinação nas áreas mais desiguais do país, conforme apontado no presente estudo, pode indicar que a expansão das equipes de Estratégia de Saúde da Família (ESF) nas áreas mais pobres do país, em especial nas regiões Norte e Nordeste, pode ter contribuído para que as ações de prevenção e promoção à saúde tenham chegado nestas áreas mais vulneráveis.

Um estudo atual sobre cobertura vacinal na América Latina e no Caribe mostrou que as taxas de vacinação são inferiores às definidas pela Organização Pan-Americana da Saúde. Fatores socioeconômicos adversos, baixo nível de escolaridade, falta de conscientização sobre doenças e suas vacinas, crenças religiosas e culturais são obstáculos frequentes à aceitação da vacinação. Contudo, níveis mais altos de educação e confiança nos profissionais de saúde, bem como a inclusão de programas educacionais para famílias, favorecem a aceitação da vacina e, portanto, devem ser considerados na implementação de estratégias para aumentar a adesão à vacinação ${ }^{23}$.

Percebe-se que a resistência à vacina não é um problema apenas do Brasil. Em vários países observa-se uma cobertura aquém do esperado, como é o caso de Uruguai e Peru. O Uruguai possui a menor taxa de cobertura vacinal da América Latina, devido à abordagem utilizada no programa de imunização, e o Peru, ainda sofre com a escassez de profissionais ${ }^{24}$.

O presente estudo apresenta algumas limitações, como o viés de memória, pois alguns 
questionamentos necessitam de dados contidos na memória dos adolescentes. Também deve-se reconhecer que adolescentes tendem a não responder com fidelidade a perguntas relacionadas a essa temática. Os resultados do presente estudo estão limitados à amostra pesquisada tendo em vista que a modelagem estatística aplicada não considerou os pesos amostrais de cada participante, o que impossibilita a generalização dos resultados para os escolares brasileiros.

Entretanto, pelo tamanho da amostra e pelas características da amostragem da PeNSE 2015, as informações referidas neste estudo são válidas e úteis, pois os resultados desse inquérito podem, em maior medida, refletir o panorama brasileiro e fornecer informação valiosa para embasar a organização de políticas públicas voltadas à minimização do problema aqui apontado, além de servir de comparação com o de outros países.

Nossos resultados refletem a necessidade de um aperfeiçoamento do sistema de saúde brasileiro com vistas à correção das iniquidades apontadas, e reforçamos também a necessidade do fortalecimento das políticas de promoção à saúde voltada para a população adolescente para a transformação deste panorama, além da necessidade do acompanhamento desse quadro ao longo dos anos a partir de novos inquéritos populacionais no Brasil.

Dentre as possibilidades, destacamos a relevância de estudos futuros que investiguem de forma mais minuciosa os meandros da dificuldade de acesso à informação e as formas de integração entre comunidade-serviço-escola nos espaços locorregionais em que este evento se evidencia, os quais podem trazer importantes respostas em prol de um sistema de saúde brasileiro mais equânime, resolutivo, com vistas à saúde dos grupos minoritários.

\section{Conclusão}

Os achados desse estudo indicam que o desconhecimento sobre a campanha de vacinação contra o HPV entre adolescentes avaliados na PeNSE 2015 foi associado às características individuais e do contexto da escola e da unidade de federação. Este estudo, de maneira geral, corrobora a compreensão multidimensional do acesso às ações e aos serviços de saúde e sua relação com as condições de saúde e vida da população e indica a importância de fortalecer a promoção à saúde voltada aos jovens em vulnerabilidade.
Dentre as ações de promoção e prevenção, destacamos que as ações educativas devem abordar informações quanto ao HPV, ao câncer de colo de útero, os objetivos da vacinação e seus resultados esperados, a prevenção de infecções sexualmente transmissíveis e de ações que valorizem a participação do adolescente e favoreçam sua autonomia, estimulando-os a assumirem comportamentos saudáveis. A comunicação deverá ser contínua, em linguagem clara, consistente e culturalmente adequada, direcionada aos adolescentes, à família, aos profissionais de saúde, aos professores e à população em geral, buscando informar e sensibilizar quanto ao tema.

Para garantir o acesso universal dos adolescentes brasileiros à imunização contra o HPV e o impacto na saúde das adolescentes de hoje e das mulheres no futuro, alguns obstáculos precisarão ser superados. Além da participação dos familiares e das escolas, a rede de atenção à saúde deverá garantir o acesso da população aos imunobiológicos e equipamentos para conservação de sua qualidade; capacitação de seus profissionais quanto à adequada administração de vacinas e às orientações de cuidados pós-vacinais e à observância ao cumprimento do plano de vacinação; além da oferta de oportunidades para a atualização vacinal dos suscetíveis, promovendo a proteção coletiva contra as doenças imunopreveníveis.

Além do fortalecimento das ações estratégias do Programa Nacional de Imunizações, fundamentais para que as ações de imunização tenham um impacto coletivo de prevenção das doenças imunopreveníveis, a análise do acesso, oferta e uso de serviços de saúde necessita ser complementada com avaliações sobre a qualidade do cuidado ofertado, o que demanda a abordagem de outras dimensões do desempenho do sistema de saúde, como adequação, continuidade, aceitabilidade, efetividade, eficiência, segurança e respeito aos direitos dos indivíduos.

\section{Colaboradores}

MAP Santos e FCGM Fernandes colaboraram na redação do artigo. KC Lima colaborou na pesquisa, análise e interpretação dos dados. IR Barbosa concebeu e planejou o estudo, colaborou na redação do artigo, e aprovou a versão final. 


\section{Referências}

1. Interaminense INCS, Oliveira SC, Leal LP, Linhares FMP, Pontes CM. Tecnologias educativas para promoção da vacinação contra o papilomavírus humano: revisão integrativa da literatura. Texto Contexto Enferm 2016; 25(2):e2300015.

2. Brasil. Organização Mundial de Saúde (OMS). Folha informativa - HPV e câncer do colo do útero [Internet]. 2019 [acessado 2020 fev 6]. Disponível em: https:// www.paho.org/bra/index.php?option=com_content\&view=article\&id=5634:folha-informativa-hpv-ecancer-do-colo-do-utero\&Itemid $=839$.

3. Chan CK, Aimagambetova G, Ukybassova T, Kongrtay K, Azizan A. Human Papillomavirus Infection and Cervical Cancer: Epidemiology, Screening, and Vaccination. Review of Current Perspectives. J Oncol 2019; 2019:3257939.

4. Colpani V, Soares F, Bacelo BA, Kops NL, Falavigna M, Serpa Hammes L, Adele SB, Ana GKM, Carla MA, Eliana MW. Prevalence of human papillomavirus (HPV) in Brazil: A systematic review and meta-analysis. PLoS One 2020; 15(2):e0229154.

5. Bray F, Ferlay J, Soerjomataram I, Siegel RL, Torre LA, Jemal A. Global Cancer Statistics 2018: GLOBOCAN Estimates of incidence and mortality worldwide for 36 cancers in 185 countries. CA Cancer J Clin 2018; 68(6):394-424.

6. Brasil. Instituto Nacional do Câncer (INCA). Câncer do colo do útero - versão para Profissionais de Saúde [Internet]. 2018 [acessado 2020 fev 6]. Disponível em: https://www.inca.gov.br/tipos-de-cancer/cancer-docolo-do-utero/profissional-de-saude.

7. Alves AC. Notícias veiculadas na mídia a respeito das queixas de adolescentes relativas à reação após a vacinação contra o HPV. Unimontes Cientifica 2017; 1(19):115-121.

8. Brasil. Ministério da Saúde (MS). HPV: o qué, causas, sintomas, tratamento, diagnóstico e prevenção [Internet]. 2019 [acessado $2020 \mathrm{fev} 7$ ]. Disponível em: https://saude.gov.br/saude-de-a-z/hpv.

9. Viegas SMF, Pereira GP, Pimenta MA, Lanza MF, Oliveira $\mathrm{PP}$, Oliveira VC. Preciso mesmo tomar vacina? Informação e conhecimento de adolescentes sobre as vacinas. Av Enferm 2019; 37(2):217-226.

10. Miranda LSMV, Souza EM. Conhecimento dos adolescentes sobre métodos contraceptivos e assistência em saúde. Rev Interdiscipl Saude 2020; 7(1):775-791.

11. Silva CS, Bodstein RCA. Referencial teórico sobre práticas intersetoriais em Promoção da Saúde na Escola. Cien Saude Colet 2016; 21(6):1777-1788.

12. Dias TS, Munhoz TN. Percepção de cuidado familiar e bullying em escolares brasileiros: Pesquisa Nacional de Saúde do Escolar (PeNSE, 2015). Lat Am J Develop 2019; 1(1):30-35.

13. Instituto Brasileiro de Geografia e Estatística (IBGE). Pesquisa Nacional de Saúde do Escolar (PeNSE) 2015. Rio de Janeiro: IBGE; 2016.

14. Malta DC, Antunes JT, Prado RR, Assunção AA, Freitas MI. Fatores associados aos episódios de agressão familiar entre adolescentes, resultados da Pesquisa Nacional de Saúde do Escolar (PeNSE). Cien Saude Colet 2019; 24(4):1287-1298.
15. Oliveira MM, Campos MO, Andreazzi MAR, Malta DC. Características da Pesquisa Nacional de Saúde do Escolar - PeNSE. Epidemiol Serv Saude 2017; 26(3):605-616.

16. Mello FCM, Silva JLD, Oliveira WAD, Prado RRD, Malta DC, Silva MAI. A prática de bullying entre escolares brasileiros e fatores associados, Pesquisa Nacional de Saúde do Escolar 2015. Cien Saude Colet 2017; 22(9):2939-2948.

17. Furlanetto MF, Marin AH, Gonçalves TR. Acesso e qualidade da informação recebida sobre sexo e sexualidade na perspectiva adolescente. Estud Pesqui Psicol 2019; 19(3):644-664

18. Santos ACD, Gasparim CA, Monteiro GM, Brito MR, Silva VAM. Relato de Experiência: construção e desenvolvimento do Programa de Saúde na Escola (PSE) sob a perspectiva da sexualidade na adolescência. Rev Bras Educ Med 2019; 43(4):193-199.

19. Azevedo F, Esteva L, Ferreira CP. Assessing the Impact of Prophylactic Vaccines on HPV Prevalence. TEMA 2019; 20(2):305-321.

20. Felisbino-Mendes MS, Paula TF, Machado IE, Oliveira-Campos M, Malta DC. Análise dos indicadores de saúde sexual e reprodutiva de adolescentes brasileiros, 2009, 2012 e 2015. Rev Bras Epidemiol 2018; 21(Supl. 1):E180013.supl.1.

21. Sousa PDL, Takiuti AD, Baracat EC, Sorpreso ICE, Abreu LC. Knowledge and acceptance of HPV vaccine among adolescents, parents and health professionals: construct development for collection and database composition. J Hum Growth Dev 2018; 28(1):58-68.

22. Abreu MNS. Conhecimento e percepção sobre o HPV na população com mais de 18 anos da cidade de Ipatinga, MG, Brasil. Cien Saude Colet 2018; 23(3):849860 .

23. Guzman-Holst A, DeAntonio R, Prado-Cohrs D, Juliao P. Barriers to vaccination in Latin America: A systematic literature review. Vaccine 2020; 38(3):470-481.

24. Restrepo FLH, Guzman, NA, Gomez ALH, Ruiz C. Policies and processes for human papillomavirus vaccination in Latin America and the Caribbean. Rev Panam Salud Publica 2017; 41:e124.

Artigo apresentado em 22/03/2020

Aprovado em 23/10/2020

Versão final apresentada em 25/10/2020

Editores-chefes: Romeu Gomes, Antônio Augusto Moura da Silva 